\title{
Cinétique de la dégradation dans le rumen de la matière sèche et de l'azote de graines de légumineuses méditerranéennes
}

\author{
CM Guedes, A Dias da Silva \\ Universidade de Trás-os-Montes e Alto Douro, Departamento de Zootecnia, \\ Apartado 202, 5001 Vila Real Codex, Portugal
}

(Reçu le 23 février 1995 ; accepté le 29 avril 1996)

\begin{abstract}
Résumé - Trois vaches adultes munies d'une canule du rumen ont été utilisées pour étudier, par la méthode des sachets de nylon, la cinétique de dégradation dans le rumen de 8 graines de légumineuses: gesse chiche (Lathyrus cicera), gesse ocre (Lathyrus ochrus), lupin (Lupinus angustifolius), pois (Pisum sativum), vesce à grands fruits (Vicia macrocarpa), vesce à fleurs isolées (Vicia monanthus), vesce de Narbonne (Vicia narbonensis) et vesce commune (Vicia sativa). Un échantillon de tourteau de soja a également été étudié. Les temps d'incubation ont été de $0,3,6,9,12,18$ et 24 heures. L'ajustement des cinétiques de dégradation a été fait par l'équation exponentielle $p=a+b\left(1-e^{-c(t-t)}\right)$ où $p$ est la fraction dégradée pendant le temps $t$, a est la fraction rapidement dégradable, $b$ est la fraction plus lentement dégradable, $c$ est la vitesse de dégradation, $t$ est le temps d'incubation et $t$ est le temps de latence. La dégradabilité théorique (DT) a été calculée par l'équation $D T=a+b c e^{-k t / 1}(c+k)$ où $k$ est le taux de sortie des particules hors du rumen. Pour un taux de sortie des particules hors du rumen de $4,4 \% \mathrm{~h}^{-1}$ la DT de l'azote a varié de $79,6 \%$ (vesce à fleurs isolées) à $87,8 \%$ (pois), ce qui montre que toutes les graines de légumineuses étudiées sont des sources protéiques de dégradabilité dans le rumen très élevée. Pour le même taux de sortie des particules hors du rumen, la DT de la MS de ces graines a varié de $71 \%$ (vesce de Narbonne) à $80,4 \%$ (pois), ce qui démontre aussi que l'énergie de ce groupe d'aliments est très fermentescible dans le rumen.
\end{abstract}

dégradabilité / matière sèche / azote / graines de légumineuses

Summary - Ruminal dry matter and crude protein degradability of Mediterranean legume seeds. Three mature cows fitted with permanent rumen cannulae and fed at maintenance level a forage:concentrate diet (75:25) were used to measure the extent and rate of degradation of dry matter (DM) and nitrogen $(\mathrm{N})$ in ground $(4 \mathrm{~mm}$ ) seeds of eight different legume species: dwarf chicklingvetch (Lathyrus cicera), ochrus vetch (Lathyrus ochrus), lupin (Lupinus angustifolius), pea (Pisum sativum), big seeded vetch (Vicia macrocarpa), bard vetch (Vicia monanthos), Narbonne vetch (Vicia narbonensis) and common vetch (Vicia sativa). A sample of soya bean meal was also used. The bags were suspended in the rumen for $0,3,6,9,12,18$ and $24 \mathrm{~h}$. Losses from the bags were estimated as time $0 \mathrm{~h}$. The kinetics of $D M$ and $N$ disappearence were studied by fitting the data to the equation 
$\mathrm{p}=\mathrm{a}+\mathrm{b}\left(1-\mathrm{e}^{-\mathrm{c}(t-t)}\right)$, where $\mathrm{p}=$ the actual degradation after time $\mathrm{t}, \mathrm{a}=$ the intercept of the degradation curve at time zero, $\mathrm{b}=$ the fraction that will be degraded when given sufficient time for digestion in the rumen, $\mathrm{c}=$ the rate constant for the degradation of fraction $\mathrm{b}, \mathrm{t}=$ the time of incubation and $\mathrm{tl}=$ the lag time. The effective degradability $(E D)$ of $D M$ and $N$ was calculated from the equation $E D=a+b c e^{-k t 1 /}(c+k)$, where $\mathrm{k}$ is the fractional oufflow rate from the rumen of feed particles. The fractional outflow rate from the rumen was calculated from the slope of the regression of logarithmic values of the decrease in chromium concentration in rumen fluid or in faeces with time. There were no significant differences $(\mathrm{P}>0.05)$ between the fractional outflow rate from the rumen obtained from rumen or faecal chromium concentration. The lowest figures for constants of DM degradation $\mathrm{a}(\%), \mathrm{b}(\%), \mathrm{c}\left(\mathrm{h}^{-1}\right)$ and $\mathrm{tt}(\mathrm{h})$, were $32.4,48.7$, 0.131 and 1.5 for bard vetch, ochrus vetch, pea and lupin, respectively, while the highest figures were $41.1,66.8,0.278$ and 4.1 for ochrus vetch, lupin, bard vetch and dwarf chicklingvetch, respectively. The corresponding figures for $N$ were 31.1, 48.5, 0.165 and 0.8 for bard vetch, pea, ochrus vetch and lupin, respectively, and 51.5, 66.2, 0.312 and 4.4 for pea, common vetch, bard vetch and dwarf chicklingvetch, respectively. The potentially degradable fraction $(\mathrm{a}+\mathrm{b})$ of $\mathrm{N}$ was very close to $100 \%$ for all legume seeds and soya bean meal, while DM potentially degradable into the rumen was 5 to 10 points lower except for pea, lupin and soya bean meal which reached 100\%. For an observed fractional outflow rate from the rumen of $4.4 \% \mathrm{~h}^{-1}$, the ED of DM varied from $71.0 \%$ (Narbonne vetch) to $80.4 \%$ (pea) while for $N$ it ranged from $79.6 \%$ (bard vetch) to $87.8 \%$ (pea). The corresponding figures for soya bean meal were 78.9 and $74.6 \%$. We conclude that these legume seeds are mainly a source of fermentable energy and degradable nitrogen for rumen microbial growth.

ruminal degradation / dry matter / nitrogen / Mediterranean legume seeds

\section{INTRODUCTION}

La politique agricole commune a encouragé pendant des années la production de sources protéiques pour l'alimentation animale avec l'objectif de réduire le déficit en protéine dans la Communauté européenne.

L'effet de cette politique s'est traduit par une augmentation de la production de pois, de fève et de féverole essentiellement en Europe du Nord. Or, il existe en Europe du Sud, et particulièrement dans les régions méditerranéennes, des légumineuses présentant un intérêt dans les systèmes d'agriculture extensive. Traditionnellement, ces graines de légumineuses sont utilisées dans l'alimentation animale, mais à l'exception du pois, du lupin et de la fève, on ne connaît pas les caractéristiques nutritionnelles de légumineuses telles que les gesses et les vesces pour les ruminants.

En effet, la plupart des travaux ont jusqu'à présent mesuré la valeur de remplacement de la fraction proteique (Cronjé, 1983; Illg et al, 1987; Guillaume et al, 1987) et sa dégradation dans le rumen (Freer et
Dove, 1984; Valentine et Bartsch, 1987; Aguilera et al, 1992; Castrillo et al, 1992), ou étudié la digestibilité totale (Hadjipanayiotou et al, 1985) et les facteurs anti-nutritionnels (Wiseman et Cole, 1988; Huisman et Tolman, 1992).

Ce travail a pour but de décrire la cinétique de dégradation dans le rumen de la matière sèche et de la fraction protéique de quelques graines de légumineuses méditerranéennes en utilisant la méthode des sachets de nylon.

\section{MATÉRIEL ET MÉTHODES}

\section{Récolte et préparation des échantillons}

L'étude a porté sur huit échantillons de graines de légumineuses, fournies par la Station nationale d'amélioration des plantes (Elvas) : gesse chiche (Lathyrus cicera cv Elbro 8987), gesse ocre (Lathyrus ochrus cv 8786), lupin (Lupinus angustifolius cv 8176), pois (Pisum sativum cv 4740), vesce à grands fruits (Vicia macrocarpa cv inconnu), vesce à fleurs isolées (Vicia monan- 
thos cv inconnu), vesce de Narbonne (Vicia narbonensis cv 8977) et vesce commune (Vicia sativa cv Gil Vaz 8773 ). Un échantillon de tourteau de soja deshuilé a aussi été étudié.

Les échantillons ont été broyés à la grille de $4 \mathrm{~mm}$. Un échantillon a été prélevé pour l'incubation dans le rumen. Le reste a été séché à $65^{\circ} \mathrm{C}$ pendant 24 heures à l'étuve avec ventilation forcée pour la détermination de la teneur en matière sèche (MS) et a ensuite été broyé à la grille de $1 \mathrm{~mm}$ pour les analyses chimiques.

L'indice d'uniformité (IU) et le module de finesse (MF) ont été mesurés sur les échantillons broyés à la grille de $4 \mathrm{~mm}$ par la méthode proposé par l'American Society of Agricultural Engineers (Asae, 1969). La granulométrie des aliments a été déterminée par tamisage en milieu $\mathrm{sec}$, pendant 10 minutes, sur huit tamis de maille $9,51,4,76,2,38 \mathrm{~mm}$ (particules grossières), 1,18, $0,6 \mathrm{~mm}$ (particules moyennes), $0,30,0,15$ et $0,05 \mathrm{~mm}$ (particules fines).

\section{Animaux et régimes}

Pour l'incubation des échantillons et la détermination du taux de sortie des particules hors du rumen, trois vaches porteuses d'une canule du rumen (poids vif moyen $580 \mathrm{~kg}$ ) ont été utilisées. Elles ont été logées dans des cases individuelles $(150 \times 102 \mathrm{~cm})$ et ont reçu une ration composée par $6 \mathrm{~kg}$ de foin de prairie naturelle $(10,2 \%$ MAT) haché en brins d'environ $10 \mathrm{~cm}$ et $2 \mathrm{~kg}$ d'aliment concentré $(57 \%$ d'orge $+37 \%$ de tourteau de soja $+6 \%$ d'un complément mineral et vitaminique). La ration a été distribuée en deux repas par jour à 12 heures d'intervalle ( 8 et 20 heures). Les animaux disposaient d'eau à volonté et l'essa' a commencé après une période de 2 semaines d'adaptation au régime.

\section{Détermination de la dégradabilité de la MS et de l'azote}

\section{Procédure}

La dégradabilité de la MS et de l'azote a été mesurée par la méthode des sachets de nylon (Ørskov et al, 1980). Les mesures de la dégradation ont été effectuées selon Dias-da-Silva et Guedes (1990). Les sachets ont été incubés pen- dant $3,6,9,12,18$ et 24 heures, puis rincés dans une machine à laver durant 40 minutes à $40^{\circ} \mathrm{C}$, séchés à $65^{\circ} \mathrm{C}$ pendant 24 heures et pesés pour la détermination de la MS résiduelle. L'azote sur les résidus d'incubation a été dosé pour la mesure de la quantité d'azote résiduel.

Les sachets contenant les échantillons ont été placés dans le rumen des vaches à raison de deux répétitions par vache et par temps d'incubation. Ainsi, pour chaque aliment, chaque point de la cinétique de dégradation de la MS est la moyenne de six mesures. Les dosages de l'azote sur les résidus ont été réalisés après un regroupement des sachets correspondant à une même durée d'incubation et à une même vache. Ainsi chaque point de la cinétique de dégradation de l'azote correspond à trois mesures.

\section{Détermination des pertes initiales}

Pour déterminer les pertes de MS et de $\mathrm{N}$ au temps 0 ( $t=0$ heure), deux sachets par aliment ont été soumis à un rinçage, identique à celui des sachets incubés dans le rumen (en machine pendant 40 minutes à $40^{\circ} \mathrm{C}$ ). Pour la mesure des pertes de la fraction soluble au temps 0 , un échantillon de $2 \mathrm{~g}$ d'aliment a été mis dans l'eau pendant 30 minutes, filtré et rincé à l'eau sur Whatman $n^{\circ} 1$. Le résidu et le papier ont été séchés à $60^{\circ} \mathrm{C}$, pesés et la fraction de MS soluble calculée par différence (DeB Hovell et al, 1986). De la même façon la fraction azotée soluble a été calculée par difference après dosage de l'azote dans le residu.

\section{Ajustement des données}

L'ajustement des données des cinétiques de dégradation a été fait conformément au modèle proposé par Ørskov et McDonald (1979) modifié par McDonald (1981) avec estimation simultanée des paramètres (Dhanoa, 1988):

$\begin{array}{ll}p=a & \text { pour } t \leq t / \\ p=a+b\left(1-e^{-c(t-t \mid)}\right) & \text { pour } t>t)\end{array}$

où $p$ est la dégradation de l'aliment incubé pendant le temps $t$, a est la fraction rapidement dégradable, $b$ est la fraction de dégradation plus lente, $a+b$ est la fraction potentiellement dégradable (asymptote de la courbe de dégradation), $c$ est la vitesse de dégradation horaire de la fraction $b, t$ est le temps d'incubation et $t /$ est le temps de 
latence, correspondant au temps écoulé avant le début de la dégradation de la fraction $b$.

La dégradabilité théorique (DT) a été calculée par l'équation de Ørskov et McDonald (1979) modifiée par Dhanoa et al (1995) :

$$
\mathrm{DT}=a+b c e^{-k t / /(c+k)}
$$

où $k$ est le taux de sortie des particules hors du rumen et $\mathrm{e}^{\mathrm{ktl}}$ est la correction pour les particules qui sortent du rumen pendant le temps de latence.

\section{Mesures sur les animaux}

\section{Cinétique postprandiale du $\mathrm{pH}$ et de la teneur en ammoniac du rumen}

Pour le contrôle du pH et de la teneur en ammoniac du rumen, le jus de rumen a été prélevé pendant 2 jours non consécutifs. Les prélèvements ont été pratiqués juste avant le repas du matin et $1,2,4,6,8$ et 12 heures après l'ingestion d'aliment. Le $\mathrm{pH}$ a été mesuré juste après chaque prélèvement. Le jus de rumen a été congelé à $-15^{\circ} \mathrm{C}$ pour la détermination de l'ammoniac.

\section{Taux de renouvellement de la phase solide du contenu du rumen}

Pour mesurer le taux de renouvellement de la phase solide du contenu du rumen, un échantillon de tourteau de soja a été marqué au dichromate de sodium selon la technique proposée par Eliman et Ørskov (1984a). On a utilisé $18,75 \mathrm{~g}$ de dichromate de sodium $\left(\mathrm{Na}_{2} \mathrm{Cr}_{2} \mathrm{O}_{7}\right)$ pour $100 \mathrm{~g}$ de MS d'échantillon ( $7 \mathrm{~g}$ de chrome pour $100 \mathrm{~g}$ de MS). La digestibilité in vitro du tourteau marqué (Tilley et Terry, 1963, modifié par Marten et Barnes, 1980) a été de $2,05 \pm 0,5 \%(n=5)$.

La quantité de $100 \mathrm{~g}$ de tourteau de soja marqué a été introduite dans le rumen de chaque vache. Les prélèvements ont été effectués à intervalles variables pendant 4 jours pour le rumen (19 prélèvements) et 7 jours pour les fèces (29 prélèvements).

La cinétique de décroissance des concentrations du chrome (rumen et fèces) a été ajustée au modèle exponentiel proposé par Grovum et Williams (1973).

\section{Analyses chimiques}

La teneur en cendres des échantillons de graines de légumineuses et du tourteau de soja a été déterminée par la méthode de l'AOAC (1990). Les matières azotées totales (MAT; $N \times 6,25$ ) ont été dosées par la méthode de Kjeldahl et les parois totales (NDF) par la méthode de Robertson et Van Soest (1981). L'amidon a été déterminé par polarimétrie selon la Norme portugaise pour le dosage de l'amidon (Norme portugaise, 1987) et les matières grasses (MG) ont été dosées par extraction à l'éther de pétrole pendant 6 heures dans un appareil Soxhlet (Anonyme, 1971). La concentration en azote ammoniacal du jus de rumen a été determinée par la méthode de Conway et O'Malley (1942). Enfin le chrome a été dosé par spectrophotométrie d'absorption atomique (Perkin Elmer 2380).

\section{Analyse statistique}

Pour tester l'effet de l'origine botanique des graines sur les paramètres de la cinétique de dégradation $(a, b, c, t l, \mathrm{DT})$ de la MS, une analyse de variance a été effectuée selon le modèle : $Y=$ moyenne + effet graine + effet animal + effet graine $x$ animal + résiduelle. Dans le cas de l'azote on a utilisé un modèle simplifié ( $Y=$ moyenne + effet graine + résiduelle) car les residus correspondant à une même durée d'incubation et à une même vache ont été regroupés. Enfin les moyennes ont été comparées par la plus petite différence significative.

Les valeurs du taux de sortie des particules hors du rumen ont été comparées par analyse de variance après ajustement de la cinétique de décroissance des concentrations de chrome du contenu du rumen et des fèces.

\section{RÉSULTATS ET DISCUSSION}

La composition chimique des graines de légumineuses et du tourteau de soja est rapportée au tableau I. Si l'on se réfère à la bibliographie, on constate une grande variabilité dans la composition chimique des cultivars d'une même espèce. De plus, pour un même cultivar, la composition varie en 
Tableau I. Composition chimique des graines de légumineuses et du tourteau de soja (\% MS).

\begin{tabular}{|c|c|c|c|c|c|}
\hline Aliments & MAT & $M G$ & NDF & Amidon & Cendres \\
\hline $\begin{array}{l}\text { Gesse chiche } \\
\text { ( } L \text { cicera })\end{array}$ & 22,3 & 1,2 & 40,3 & 25,3 & 3,9 \\
\hline $\begin{array}{l}\text { Gesse ocre } \\
\text { (L ochrus) }\end{array}$ & 21,8 & 1,7 & 32,9 & 27,3 & 4,2 \\
\hline $\begin{array}{l}\text { Lupin } \\
\quad \text { ( } L \text { angustifolius) }\end{array}$ & 34,2 & 7,7 & 27,1 & 1,10 & 4,0 \\
\hline $\begin{array}{l}\text { Pois } \\
(P \text { sativum })\end{array}$ & 30,1 & 2,3 & 26,4 & 36,2 & 4,0 \\
\hline $\begin{array}{l}\text { Vesce à grands fruits } \\
\text { ( } V \text { macrocarpa })\end{array}$ & 23,6 & 1,1 & 39,1 & 28,5 & 3,4 \\
\hline $\begin{array}{l}\text { Vesce à fleurs isolées } \\
\text { ( } V \text { monanthus) }\end{array}$ & 23,0 & 9,7 & 31,1 & 29,3 & 5,0 \\
\hline $\begin{array}{l}\text { Vesce de Narbonne } \\
\text { ( } V \text { narbonensis) }\end{array}$ & 22,9 & 6,8 & 38,7 & 24,1 & 3,3 \\
\hline $\begin{array}{l}\text { Vesce commune } \\
\text { ( } V \text { sativa) }\end{array}$ & 23,0 & 1,5 & 43,9 & 25,1 & 3,8 \\
\hline Tourteau de soja & 47,6 & 1,6 & 17,4 & 5,10 & 6,4 \\
\hline
\end{tabular}

fonction du stade de végétation des plantes, des techniques agronomiques et des conditions de stockage des graines (Hill, 1977 ; Newton et Hill, 1983 ; Wiseman et Cole, 1988 ; Dixon et Hosking, 1992). Parmi les graines de légumineuses étudiées, la gesse ocre a une teneur en MAT inférieure à celle trouvée par Hadjipanayiotou et al (1985) $(21,8$ vs $31,3 \%)$. À l'inverse, le pois a des teneurs en MAT $(30,1 \%)$ et en parois totales $(26,4 \%)$ supérieures à celles trouvées par différents auteurs (Lindberg, 1985 ; INRA, 1988 ; Michalet-Doreau et Cerneau, 1991 ; Castrillo et al, 1992 ; Dixon et Hosking, 1992).

Les données du tableau I ne donne qu'une information partielle sur la teneur en glucides résistants à l'hydrolyse par les enzymes d'origine animale, notamment pour le lupin et le tourteau de soja et à un moindre degré, la gesse ocre et la gesse chiche. En effet, il est bien connu que les substances pectiques et les $\beta$-galactanes présentes dans les graines (Carré et Brillouet, 1986), sont solubilisées par la solution de détergent neutre (Van Soest et al, 1991). En outre, la teneur en $\alpha$-galactosides, composées également solubles dans cette solution, peut varier de 6,7 à $12,0 \%$ de la MS pour le lupin (Trugo et al, 1988) et de 5,0 à $7,2 \%$ de la MS pour le pois (Wiseman et Cole, 1988).

Les résultats des mesures de granulométrie présentés dans le tableau II montrent que l'IU ou le MF des graines de légumineuses et du tourteau de soja sont très proches. Pour cette raison le tourteau de soja marqué a été utilisé pour estimer le taux de renouvellement des particules des graines dans le rumen en admettant que le traitement au dichromate de sodium est sans effet sur la granulométrie en accord avec Eliman et Ørskov (1984a).

Dans notre étude le taux de renouvellement observé a été de $4,4 \% \mathrm{~h}^{-1}$ en moyenne, indépendamment des vaches et du lieu des prélèvements ce qui confirme les résultats de Eliman et Ørskov (1984b) et de Vallepuga et al (1986).

Les cinétiques postprandiales du $\mathrm{pH}$ et de la teneur en ammoniac dans le rumen 
Tableau II. Granulométrie des graines de légumineuses et du tourteau de soja.

\begin{tabular}{|c|c|c|c|c|c|}
\hline Aliments & $\begin{array}{l}\text { Particules } \\
\text { grossieres } \\
(>2,38 \mathrm{~mm})\end{array}$ & $\begin{array}{c}\text { Particules } \\
\text { moyennes } \\
(2,38-0,6 \mathrm{~mm})\end{array}$ & $\begin{array}{l}\text { Particules } \\
\text { fines } \\
(<0,6 \mathrm{~mm})\end{array}$ & $U^{a}$ & $M F b$ \\
\hline $\begin{array}{l}\text { Gesse chiche } \\
\text { ( } L \text { cicera })\end{array}$ & 0,000 & 0,826 & 0,174 & $0: 8: 2$ & 3,46 \\
\hline $\begin{array}{l}\text { Gesse ocre } \\
\text { (L ochrus) }\end{array}$ & 0,000 & 0,673 & 0,327 & $0: 7: 3$ & 2,93 \\
\hline $\begin{array}{l}\text { Lupin } \\
\qquad(L \text { angustifolius })\end{array}$ & 0,000 & 0,821 & 0,179 & $0: 8: 2$ & 3,41 \\
\hline $\begin{array}{l}\text { Pois } \\
\text { (P sativum })\end{array}$ & 0,000 & 0,832 & 0,168 & $0: 8: 2$ & 3,43 \\
\hline $\begin{array}{l}\text { Vesce à grands fruits } \\
\text { ( } V \text { macrocarpa) }\end{array}$ & 0,000 & 0,715 & 0,285 & $0: 7: 3$ & 3,20 \\
\hline $\begin{array}{l}\text { Vesce à fleurs isolées } \\
\text { ( } V \text { monanthus) }\end{array}$ & 0,000 & 0,780 & 0,220 & $0: 8: 2$ & 3,13 \\
\hline $\begin{array}{l}\text { Vesce de Narbonne } \\
\text { ( } V \text { narbonensis) }\end{array}$ & 0,000 & 0,689 & 0,308 & $0: 7: 3$ & 2,83 \\
\hline $\begin{array}{l}\text { Vesce commune } \\
\text { ( } V \text { sativa })\end{array}$ & 0,000 & 0,809 & 0,190 & $0: 8: 2$ & 3,46 \\
\hline Tourteau de soja & 0,000 & 0,817 & 0,183 & $0: 8: 2$ & 3,46 \\
\hline
\end{tabular}

a Indice d'uniformité; 'b Module de finesse (ASAE, 1969)

(fig 1) montrent que les conditions pour l'activité cellulolytique et la croissance microbienne ont été satisfaisantes (Satter et Slyter, 1974 ; Mould et al, 1983).

La proportion d'azote soluble dans l'eau et les pertes d'azote lors du rinçage des sachets sont présentées au tableau III. La teneur en azote soluble en pourcentage de l'azote total a varié de $26,6 \%$ pour la vesce commune à $42,3 \%$ pour le pois. Bien que la teneur en azote soluble et les pertes d'azote lors du lavage des sachets soient positivement correlées $(r=0,945 ; p<0,001)$, les pertes physiques d'azote dans les particules qui traversent les pores des sachets au temps $t=0$ sont variables selon les aliments. Elles sont faibles chez la gesse chiche, la vesce à fleurs isolées et la vesce de Narbonne mais relativement importantes chez le lupin, le pois, la vesce à grands fruits et le tourteau de soja. En tenant compte des don- nées de la granulométrie (tableau II), il n'y a pas de relation entre le pourcentage de particules fines des échantillons et les pertes physiques. Aussi peut-on en conclure que dans les cas où les pertes sont plus élevées les particules perdues lors du rinçage sont plus riches en azote que la moyenne de l'échantillon.

Les paramètres de dégradation et la dégradabilité théorique de la MS et de l'azote sont présentés aux tableaux IV et $\mathrm{V}$, respectivement. Malgré des différences significatives entre les animaux pour les paramètres de dégradation de la MS, les données du tableau IV montrent que cet effet est quantitativement très limité.

La valeur de la fraction potentiellement dégradable $(a+b)$ de l'azote approche les $100 \%$ pour tous les aliments. En revanche, à l'exception du pois, du lupin et du tourteau de soja, la fraction potentiellement 
Tableau III. Fraction azotée soluble et pertes d'azote (totales et physiques) lors du lavage des sachels de nylon, en \% de la matière sèche total (MS) et de l'azote total (Nt).

\begin{tabular}{llccc}
\hline Aliments & Fraction & $\begin{array}{c}\text { Pertes totales } \\
\text { lors du lavage }\end{array}$ & $\begin{array}{c}\text { Pertes physiques } \\
\text { lors du lavage a }\end{array}$ \\
\hline Gesse chiche & $\mathrm{MS}$ & 12,6 & 13,3 & \\
( $L$ cicera) & $\mathrm{Nt}$ & 35,4 & 37,2 & 0,7 \\
Gesse ocre & $\mathrm{MS}$ & 14,1 & 15,8 & 1,8 \\
(L ochrus) & $\mathrm{Nt}$ & 40,4 & 45,4 & 1,7 \\
Lupin & $\mathrm{MS}$ & 16,1 & 20,9 & 5,0 \\
(L angustifolius) & $\mathrm{Nt}$ & 29,4 & 38,2 & 4,8 \\
Pois & $\mathrm{MS}$ & 20,4 & 24,9 & 8,8 \\
( $P$ sativum) & $\mathrm{Nt}$ & 42,3 & 51,7 & 4,5 \\
Vesce à grands fruits & $\mathrm{MS}$ & 14,4 & 17,5 & 9,4 \\
(V macrocarpa) & $\mathrm{Nt}$ & 38,2 & 46,3 & 3,2 \\
Vesce à fleurs isolées & $\mathrm{MS}$ & 11,3 & 12,1 & 8,1 \\
(V monanthus) & $\mathrm{Nt}$ & 30,7 & 32,9 & 0,8 \\
Vesce de Narbonne & $\mathrm{MS}$ & 11,5 & 12,3 & 2,2 \\
(V narbonensis) & $\mathrm{Nt}$ & 31,4 & 33,6 & 0,8 \\
Vesce commune & $\mathrm{MS}$ & 9,8 & 12,1 & 2,2 \\
(V sativa) & $\mathrm{Nt}$ & 26,6 & 32,9 & 2,3 \\
Tourteau de soja & $\mathrm{MS}$ & 8,7 & 15,1 & 6,3 \\
& $\mathrm{Nt}$ & 11,4 & 19,8 & 6.4 \\
& & & & 8,4 \\
\hline
\end{tabular}

a Pertes physiques $=$ pertes totales lors du lavage - fraction soluble.

dégradable de la MS reste 5 à 10 points inférieure. Toutefois, les comparaisons entre aliments font ressortir des différences sensibles soit pour la fraction a soit pour la fraction $b$. Ainsi, la valeur la plus faible $(p<0,05)$ de la fraction rapidement dégradable $a$ de la MS et de l'azote a été observée chez le tourteau de soja $(29,8$ et $21,2 \%$, respectivement). En revanche, la gesse ocre et le pois présentent les valeurs les plus élevées
Fig 1. Variation du $\mathrm{pH}(\bullet)$ et de l'azote ammoniacal $\left(\mathrm{N}-\mathrm{NH}_{3} ; \mathrm{mg} / 100 \mathrm{~mL}\right)(\mathrm{O})$ dans le rumen.

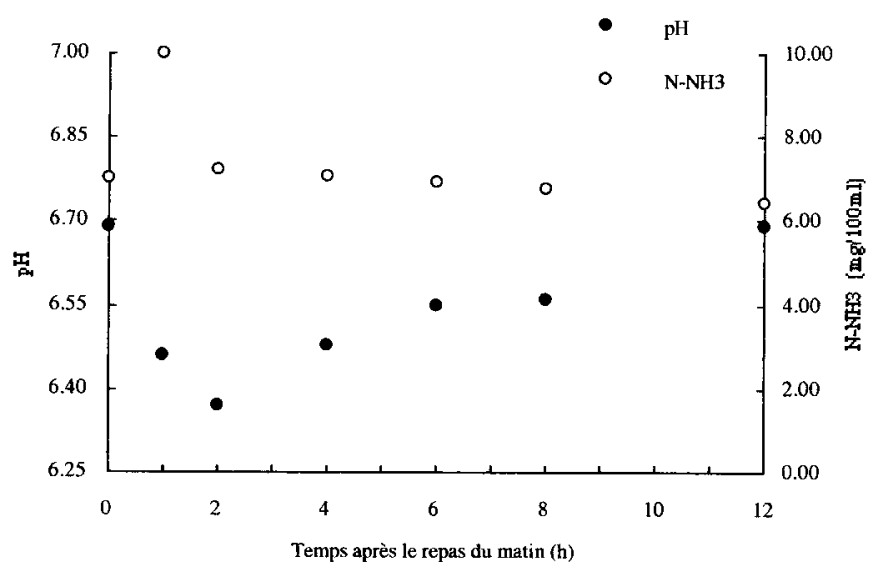


Tableau IV. Paramères de dégradation $(a, b$ et $c$ ), temps de latence ( $t$ l) et dégradabilité théorique (DT) de la MS des graines de légumineuses et du tourteau de soja.

\begin{tabular}{|c|c|c|c|c|c|}
\hline & a $(\%)$ & b (\%) & $\mathrm{c}\left(h^{-1}\right)$ & tl $(h)$ & $D T\left(k=4,4 \% h^{-1}\right)$ \\
\hline Aliments & $(n=6)$ & $(n=6)$ & $(n=6)$ & $(n=6)$ & $(n=6)$ \\
\hline $\begin{array}{l}\text { Gesse chiche } \\
\text { (L cicera })\end{array}$ & $33,5^{\mathrm{cd}}$ & $62,1^{e}$ & $0,161^{a b c}$ & $4,1^{\text {ef }}$ & $73,7^{b}$ \\
\hline $\begin{array}{l}\text { Gesse ocre } \\
\text { (L ochrus) }\end{array}$ & $41,1^{f}$ & $48,7^{a}$ & $0,192^{\mathrm{bcd}}$ & $3,7^{\text {ef }}$ & $74,1^{b}$ \\
\hline $\begin{array}{l}\text { Lupin } \\
\text { (L angustifolius) }\end{array}$ & $32,8^{b c}$ & $66,8^{f}$ & $0,135^{a b c}$ & $1,5^{\mathrm{a}}$ & $79,8^{\mathrm{e}}$ \\
\hline $\begin{array}{l}\text { Pois } \\
\text { (P sativum })\end{array}$ & $40,7^{\dagger}$ & $58,9^{d}$ & $0,131 a b$ & $2,4^{b c}$ & $80,4^{e}$ \\
\hline $\begin{array}{l}\text { Vesce à grands fruits } \\
\text { (V macrocarpa) }\end{array}$ & $39,2^{e}$ & $54,6^{\mathrm{b}}$ & $0,162^{\mathrm{abc}}$ & $3,3^{\mathrm{de}}$ & $76,3^{c}$ \\
\hline $\begin{array}{l}\text { Vesce à fleurs isolées } \\
\text { (V monanthus) }\end{array}$ & $32,4^{b}$ & $57,7^{\mathrm{c}}$ & $0,278^{d}$ & $3,6^{\mathrm{ef}}$ & $74,0^{\mathrm{b}}$ \\
\hline $\begin{array}{l}\text { Vesce de Narbonne } \\
\text { (V narbonensis) }\end{array}$ & $34,3^{d}$ & $54,4^{b}$ & $0,144^{a b c}$ & $2,7^{\text {cde }}$ & $71,0^{\mathrm{a}}$ \\
\hline $\begin{array}{l}\text { Vesce commune } \\
\text { ( } V \text { sativa) }\end{array}$ & $33,9^{c}$ & $56,3^{c}$ & $0,231^{c d}$ & $3,6^{e t}$ & $73,6^{\mathrm{b}}$ \\
\hline Tourteau de soja & $29,8^{a}$ & $70,2^{9}$ & $0,142^{\mathrm{abc}}$ & $2,0^{a b}$ & $78,9^{d}$ \\
\hline Animaux & $(n=18)$ & $(n=18)$ & $(n=18)$ & $(n=18)$ & $(n=18)$ \\
\hline 1 & $35,5^{b}$ & $58,7^{a b}$ & $0,171^{\mathrm{ab}}$ & $3,3^{b}$ & $76,1^{b}$ \\
\hline 2 & $35,4^{b}$ & $58,3^{a}$ & $0,200^{\mathrm{b}}$ & $3,2^{b}$ & $75,7^{\mathrm{ab}}$ \\
\hline 3 & $34,9^{a}$ & $59,4^{b}$ & $0,154^{a}$ & $2,4^{a}$ & $75,4^{a}$ \\
\hline \multicolumn{6}{|l|}{ Seuil de signification } \\
\hline Aliments & 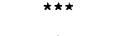 & $* * \star$ & 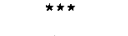 & $\star \star \star \star$ & 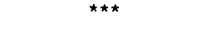 \\
\hline Animaux & * & * & $\star \star$ & $\star \star \star \star ~$ & * \\
\hline Aliments $x$ animaux & * & $\star \star \star ~$ & $* *$ & ns & ns \\
\hline ET aliments & 0,298 & 0,520 & 0,018 & 0,239 & 0,311 \\
\hline ET animaux & 0,170 & 0,300 & 0,011 & 0,138 & 0,180 \\
\hline
\end{tabular}

${ }^{* \star *} p<0,001 ;{ }^{* *} p<0,01 ;{ }^{*} p<0,05 ;$ ns: : non significatif $(p>0,05)$. Sur une colonne, les moyennes affectées d'une même lettre ne sont pas significativement différentes au seuil de $5 \%$. 
pour la MS $(41,1$ et $40,7 \%$, respectivement) et le pois la valeur la plus élevée pour l'azote $(51,5 \%)$.

La fraction plus lentement dégradable $b$ est d'autant plus élevée que la fraction a est plus faible. Cette relation inverse est très importante pour l'azote $(r=-0,995$; $p<0,001)$ mais plus faible pour la MS $(r=-0,714 ; p<0,05)$, ce qui s'explique par le fait que la fraction potentiellement dégra- dable de la MS reste pour quelques aliments (vesce commune, vesce à fleurs isolées et gesse ocre, notamment) nettement inférieure à $100 \%$. Le tourteau de soja présente la valeur de $b$ la plus élevée $(p<0,05)$ pour la MS $(70,2 \%)$ et pour l'azote $(78,4 \%)$. Parmi les graines de légumineuses, les valeurs les plus élevées sont observées chez le lupin pour la MS $(66,8 \%)$ et chez la vesce à fleurs isolées, vesce de Narbonne

Tableau V. Paramètres de dégradation ( $\mathrm{a}, \mathrm{b}$ et $\mathrm{c}$ ), temps de latence $(\mathrm{t} l)$ et dégradabilité théorique (DT) de l'azote des graines de légumineuses et du tourteau de soja.

\begin{tabular}{|c|c|c|c|c|c|}
\hline Aliments & $\begin{array}{l}a(\%) \\
(n=3)\end{array}$ & $\begin{array}{l}b(\%) \\
(\mathrm{n}=3)\end{array}$ & $\begin{array}{l}c\left(h^{-1}\right) \\
(n=3)\end{array}$ & $\begin{array}{c}t l(h) \\
(n=3)\end{array}$ & $\begin{array}{l}D T(\%) \\
(n=3)\end{array}$ \\
\hline $\begin{array}{l}\text { Gesse chiche } \\
\text { ( } L \text { cicera })\end{array}$ & $38,9^{e}$ & $60,6^{c}$ & $0,200^{\mathrm{a}}$ & $4,4^{\text {de }}$ & $\begin{array}{c}\left(\mathrm{k}=4,4 \% \mathrm{~h}^{-1}\right) \\
79,8^{\mathrm{b}}\end{array}$ \\
\hline $\begin{array}{l}\text { Gesse ocre } \\
\text { (L ochrus })\end{array}$ & $45,4^{f}$ & $54,6^{\mathrm{b}}$ & $0,165^{a}$ & $2,2^{b c}$ & $84,5^{\mathrm{cd}}$ \\
\hline $\begin{array}{l}\text { Lupin } \\
\qquad(L \text { angustifolius) }\end{array}$ & $37,7^{e}$ & $62,3^{c}$ & $0,166^{a}$ & $0,8^{a}$ & $85,2^{d}$ \\
\hline $\begin{array}{l}\text { Pois } \\
\text { ( } P \text { sativum })\end{array}$ & $51,5 \mathrm{~g}$ & $48,5^{\mathrm{a}}$ & $0,180^{\mathrm{a}}$ & $1,6^{a b}$ & $87,8^{e}$ \\
\hline $\begin{array}{l}\text { Vesce à grands fruits } \\
\text { ( } V \text { macrocarpa) }\end{array}$ & $46,5^{\dagger}$ & $53,4^{b}$ & $0,166^{a}$ & $2,8 \mathrm{~cd}$ & $83,7^{c}$ \\
\hline $\begin{array}{l}\text { Vesce à fleurs isolées } \\
\text { ( } V \text { monanthus) }\end{array}$ & $31,1^{b}$ & $66,0^{d}$ & $0,312^{b}$ & $3,8^{\text {de }}$ & $79,6^{b}$ \\
\hline $\begin{array}{l}\text { Vesce de Narbonne } \\
\text { (V narbonensis) }\end{array}$ & $33,6^{d}$ & $66,2^{\mathrm{d}}$ & $0,181^{a}$ & $1,3^{a b}$ & $83,8^{c}$ \\
\hline $\begin{array}{l}\text { Vesce commune } \\
\text { ( } V \text { sativa) }\end{array}$ & $32,9 c$ & $66,2^{d}$ & $0,173^{a}$ & $2,2^{b}$ & $80,8^{b}$ \\
\hline Tourteau de soja & $21,2^{\mathrm{a}}$ & $78,4^{\ominus}$ & $0,195^{a}$ & $3,7^{\mathrm{de}}$ & $74,6^{a}$ \\
\hline Seuil de signification & 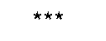 & $\star \star \star$ & * & $\star \star \star$ & *** \\
\hline ET & 0,563 & 0,854 & 0,026 & 0,364 & 0,485 \\
\hline
\end{tabular}

${ }^{\star \star \star} p<0,001 ;{ }^{*} p<0,05 ;$ ns : non significatif $(p>0,05)$. Sur une colonne, les moyennes affectées d'une même lettre ne sont pas significativement différentes au seuil de $5 \%$. 
et vesce commune, pour l'azote $(66 \%$ environ). La gesse ocre et le pois présentent les valeurs les plus faibles $(p<0,05)$ pour la MS et l'azote, 48,7 et $48,5 \%$, respectivement.

Bien que la vitesse de dégradation $c$ de la fraction $b$ de la MS soit variable entre aliments, les valeurs ne sont pas significativement différentes dans la plupart des cas. La vesce à fleurs isolées, a la vitesse de dégradation la plus élevée $\left(0,278 \mathrm{~h}^{-1}\right)$ valeur qui n'est cependant pas significativement différente de celles observées avec la vesce commune et la gesse ocre $(0,231$ et $0,192 \mathrm{~h}^{-1}$, respectivement).

La vesce à fleurs isolées présente une vitesse de dégradation $c$ de l'azote significativement supérieure $(p<0,05)$ aux autres aliments (tableau $V$ ). Pour toutes les graines sauf la vesce commune et la gesse ocre, la vitesse de dégradation de la fraction $b$ de l'azote est plus élevée que celle de la MS (tableaux IV et V).

Pour tous les aliments la méthode d'ajustement des données des cinétiques de dégradation met en évidence un temps de latence sur la dégradation de la MS et de l'azote. Les valeurs les plus faibles sont trouvées pour le lupin $(1,5$ et 0,8 heure pour la MS et l'azote, respectivement) et les valeurs les plus élevées chez la gesse chiche $(4,1$ et 4,4 heures pour la MS et l'azote, respectivement). Bien que le calcul des temps de latence aurait été amelioré avec des temps d'incubation entre 0 et 3 heures, la figure 2 montre qu'il y a un décalage entre la libération de la fraction rapidement dégradable et le début de la fermentation de la fraction la plus lentement dégradable, même quand le t/a été le plus court (lupin).

Les données de la DT, calculées à partir de l'équation [3] pour le taux de renouvellement mesuré dans cette étude $\left(4,4 \% \mathrm{~h}^{-1}\right)$, rapportées aux tableaux IV et $\mathrm{V}$ montrent que pour la MS la valeur DT varie de $71 \%$ pour la vesce de Narbonne à $80 \%$ environ pour le pois et le lupin. En ce qui concerne l'azote les valeurs extrêmes ont été observées pour le tourteau de soja $(74,6 \%)$ et le pois $(87,8 \%)$.

La comparaison de nos données de dégradation avec les résultats d'autres auteurs est difficile. En effet, à l'exception du lupin et du pois, il y a très peu d'information sur la cinétique de la dégradation des graines de légumineuses. D'autre part, les différences dans la technique des sachets de nylon (gra-

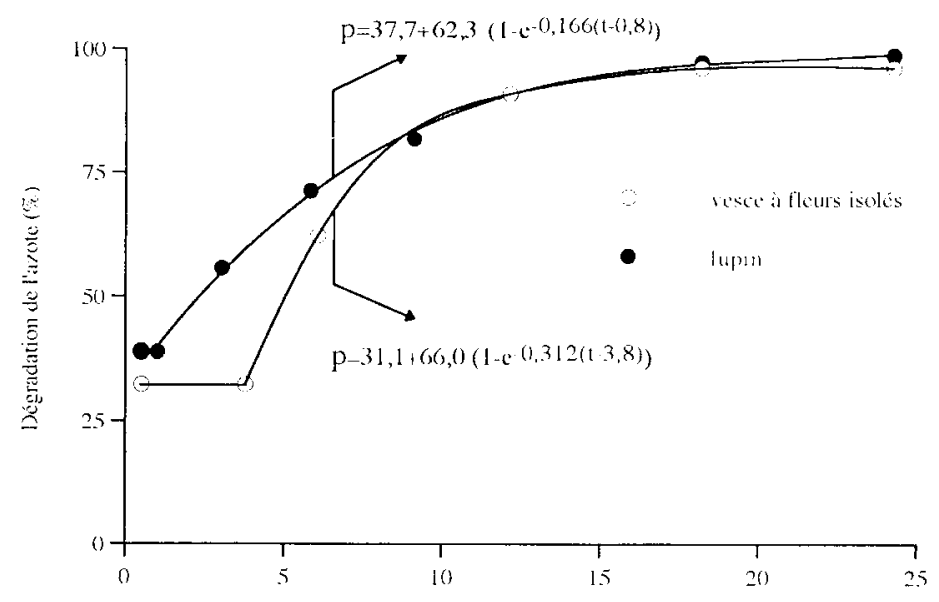

Fig 2. Courbes de dégradation de l'azote dans le rumen dans deux cas extrêmes: - lupin et $O$ vesce à fleurs isolées. 
nulométrie des échantillons, taille des pores, nombre de temps d'incubation, interprétation des données) rendent difficile la comparaison des paramètres de dégradation (Lindberg, 1985 ; Nocek, 1988; MichaletDoreau et Ould Bah, 1992 ; AFRC, 1993).

En ce qui concerne le lupin et le pois les valeurs de la somme des fractions $a$ et $b$ de l'azote rapportées dans la bibliographie sont toujours très proches de $100 \%$ malgré des différences sensibles pour les valeurs de chaque paramètre. C'est surtout pour la vitesse de dégradation de la fraction $b$ qu'on trouve les valeurs les plus variables. En effet, pour le lupin, les valeurs extrêmes 0,071 et $0,64 \mathrm{~h}^{-1}$ sont respectivement présentées par Freer et Dove (1984) et Valentine et Bartsch (1988). Enfin, pour le pois, on trouve des valeurs de 0,0895 et $0,194 \mathrm{~h}^{-1}$ (Van Straalen et Tamminga, 1990 ; Michalet-Doreau et Cerneau, 1991).

Dans le seul travail publié sur la dégradation de la vesce commune dans le rumen, Aguilera et al (1992) ont obtenu une vitesse de dégradation de la MS et de l'azote de 0,048 et $0,043 h^{-1}$, respectivement, avec des échantillons broyés à la grille de 2,0 $\mathrm{mm}$. Les fractions rapidement dégradables de la MS et de l'azote ont été, respectivement, d'environ 20 et 10 unités inférieures à nos résultats.

\section{CONCLUSIONS}

Les graines de légumineuses que nous avons étudiées, ont une dégradabilité et une vitesse de dégradation de la MS et de l'azote dans le rumen très élevée. Pour un taux de sortie des particules hors du rumen de $4,4 \% \mathrm{~h}^{-1}$, les valeurs moyennes calculées de la DT de la MS et de l'azote ont été de 75,4 et $83,2 \%$, respectivement. D'un point de vue nutritionnel, ce groupe d'aliments se caractérise comme étant des sources d'énergie et d'azote essentiellement dégradables dans le rumen.
Pour la plupart des espèces étudiées il n'y a pas d'information sur leur cinétique de dégradation dans le rumen. Pour le pois et le lupin les données disponibles sont très variables. II apparaît donc nécessaire de standardiser la technique des sachets de nylon et d'utiliser un modèle mathématique qui puisse décrire la cinétique de dégradation avec précision. Dans cette étude, le modèle de Ørskov et McDonald (1979) avec l'incorporation d'un temps de latence s'est montré parfaitement adapté à nos données.

\section{REMERCIEMENTS}

Les auteurs remercient le Conseil national de recherche scientifique (JNICT, Lisbonne) et l'Agence international d'énergie atomique (Vienne) de l'aide financière apportée à la réalisation de cette étude. Nous tenons aussi à remercier la Station nationale d'amélioration des plantes (Elvas) qui nous a offert les graines et $\mathrm{J}$ Potes pour la canulation des animaux.

\section{RÉFÉRENCES}

Agricultural and Food Research Council (1993) Technical Committee on Responses to Nutrients. Energy and Protein Requirements of Ruminants, AFRC, $\mathrm{CAB}$ International, Wallingford, Oxon, $160 \mathrm{p}$

Aguilera JF, Bustos M, Molina E (1992) The degradability of legume seed meals in the rumen: effect of heat treatment. Anim Feed Sci Techno/ 36, 101-112

American Society of Agricultural Engineers (1969) ASAE Recommendation: ASAE R246. 1. Method of determining modulus of uniformity and modulus of fineness of ground feed. Agr Eng Yearbook, 348

Anonyme (1971) Dosage des matières grasses brutes. $J$ Off Comm Europ n ${ }^{\circ}$ L 279/17, 71.12.20

Association of Official Analytical Chemists (1990) Official methods of analysis. 14th ed, Vol 1. AOAC, Washington, DC, Etats-Unis, $684 \mathrm{p}$

Carré B. Brillouet JM (1986) Yield and composition of cell wall residues isolated from various feedstuffs used for non-ruminant farm animals. $J$ Sci Food Agric 37, 341-351

Castrillo C, Lainez M, Gasa J, Guada JA (1992) The effect of increasing the proportion of barley straw in pelleted concentrate diets given to lambs on rumen 
outflow rate and degradation of protein supplements. Anim Prod 54, 59-66

Conway EJ, O'Malley F (1942) Micro-diffusion methods. Ammonia and urea using buffer absorbents. Bioch $J$ $36,655-661$

Cronjé PB (1983) Protein degradability of several South African feedstuffs by the artificial fibre bag technique. $S$ Afr J Anim Sci 13, 225-228

DeB Hovell FD, Ngambi JWW, Barber WP, Kyle DJ (1986) The voluntary intake of hay by sheep in relation to its degradability in the rumen as measured in nylon bags. Anim Prod 42, 111-118

Dhanoa MS (1988) On the analysis of dracon bag data for low degradability feeds. Grass For Sci 43, 441444

Dhanoa MS, France J, Siddons RC, Lopez S, Buchanan-Smith JG (1995) A non-linear compartmental model to describe forage degradation kinetics during incubation in polyester bags in the rumen. Br J Nutr 73, 3-15

Dias-da-Silva AA, Guedes C (1990) Variability in the nutritive value of straw cultivars of wheat, rye and triticale and response to urea treatment. Anim Feed Sci Technol 28, 79-89

Dixon RM, Hosking BJ (1992) Nutritional value of grain legumes for ruminants. Nutr Res Rev 5, 19-43

Eliman ME, Ørskov ER (1984a) Factors affecting the outflow of protein supplements from the rumen. 1. Feeding level. Anim Prod 38, 45-51

Eliman ME, Ørskov ER (1984b) Estimation of rates of outflow of protein supplements from the rumen by determining the rate of excretion of chromium-treated protein supplements in faeces. Anim Prod 39, 77-80

Freer M, Dove H (1984) Rumen degradation of protein in sunflower meal, rapeseed meal and lupin seed placed in nylon bags. Anim Feed Sci Technol 11, 87-101

Grovum WL, Williams VJ (1973) Rate of passage of digesta in sheep. 3. Differential rates of passage of water and dry matter from the reticulum-rumen, abomasum and caecum and proximal colon. Br J Nutr $30,231-240$

Guillaume B, Otterby DE, Linn JC, Stern MD, Johnson DG (1987) Comparison of sweet white lupin seeds with soybean meal as a protein supplement for lactating cows. J Dairy Sci 70, 2339-2348

Hadjipanayiotou M, Economides S, Koumas A (1985) Chemical composition, digestibility and energy content of leguminous grain and straws grown in a Mediterranean region. Ann Zootech 30, 23-30

Hill GD (1977) The composition and the nutritive value of lupin seed. Nutr Abs Rev series B 47, 511-529

Huisman Z, Tolman GH (1992) Antinutritional factors in the plant proteins of diets for non-ruminants. in: Recent Advances in Animal Nutrition (PC Garnsworthy, $\mathrm{N}$ Haresign, DJA Cole, eds), ButterworthHeinemann Ltd, Oxford, Royaume-Uni, 3-31
IIIg DJ, Sommerfeldt JL, Boe AA (1987) Chickpeas as a substitute for corn and soybean meal in growing heifer diets. J Dairy Sci 70, 2181-2185

Inra (1988) Alimentation des bovins, ovins et caprins ( $R$ Jarrige, ed), Inra, Paris, 471p

Lindberg JE (1985) Estimation of rumen degradability of feed proteins with the in sacco technique and various in vitro methods: a review. Acta Agric Scand suppl 25, 64-97

Marten GC, Barnes RF (1980) Prediction of energy digestibility of forages with in vitro rumen fermentation and fungal enzyme systems. In: Standardization of Analytical Methodology for Feeds (WJ Pigden, CC Balch, M Graham, eds), IDRC, Ottawa, ON. Canada, 61-71

McDonald I (1981) A revised model for the estimation of protein degradability in the rumen. J Agric Sci 96 , 251-252

Michalet-Doreau B, Cerneau P (1991) Influence of foodstuffs particle size on in situ degradation of nitrogen in the rumen. Anim Feed Sci Technol 35, 69-81

Michalet-Doreau B, Ould-Bah MY (1992) In vitro and in sacco methods for the estimation of dietary nitrogen degradability in the rumen: a review. Anim Feed Sci Technol 40, 57-86

Mould FL, Ørskov ER, Mann SO (1983) Associative effects of mixed feeds. 1. Effects of type and level of supplementation and the influence of the rumen fluid $\mathrm{pH}$ on cellulolysis in vivo and dry matter digestion of various roughages. Anim Feed Sci Technol 10, 15-30

Newton SD, Hill GD (1983) Composition and nutritive value of field beans. Nutr Abs rev series B 53, 99-115

Nocek JE (1988) In situ and other methods to estimate ruminal protein and energy digestibility: a review. $J$ Dairy Sci 71, 2051-2069

Norme portugaise (1987) Alimentos para animais. Determinação do teor em amido. Método polarimétrico. NP 2026, $7 p$

Orskov ER, McDonald I (1979) The estimation of protein degradability in the rumen from incubation measurements weighed according to the rate of passage. $J$ Agric Sci 92, 449-503

Ørskov ER, DeB Hovell FD, Mould F (1980) The use of the nylon bag technique for the evaluation of feedstuffs. Trop Anim Prod 5, 195-213

Robertson JB, Van Soest PJ (1981) The detergent system of analysis and its application to human food. In: The Analysis of Dietary Fiber in Food (WPT James, O Theander, eds), Marcell Deker, New York, États-Unis, 123-158

Satter LD, Slyter LL (1974) Effect of ammonia concentration on rumen microbial production in vitro. $\mathrm{Br} J$ Nutr 32, 199-208

Tilley JMA, Terry RA (1963) A two-stage technique for in vitro digestion of forage. J Br Grass Soc 18, 104111 
Trugo LC, Almeida DCF, Gross R (1988) Oligosaccharide contents in the seeds of cultivated lupins. $J \mathrm{Sci}$ Food Agric 45, 21-24

Valentine SC, Bartsch BD (1987) Fermentation of hammer milled barley, lupin, pea and faba bean grain in the rumen of dairy cows. Anim Feed Sci Technol 16, 261-271

Valentine SC, Bartsch BD (1988) Degradation of dry matter, crude protein, fat and nitrogen-free-extract in milled barley and lupin grains incubated in nylon bags in the rumen of dairy cows. J Agric Sci 110, 395-398

Vallepuga JAG, Castrillo C, Gonzalez A, Gasa GJ (1986) Rumen outflow rate estimated from decline of chromium concentration in faecal and rumen samples after dosage of sodium dichromate fish meal. Anim Prod 42, 471-472

Van Soest PJ, Robertson JB, Lewis BA (1991) Methods for dietary fiber, neutral detergent fiber and nonstarch polysaccharides in relation to animal nutrition. J Dairy Sci 74, 3583-3597

Van Straalen WM, Tamminga S (1990) Protein degradation. In: Feedstuff Evaluation. (J Wiseman, DJA Cole, eds), Butterworths, Londres, Royaume-Uni, 55-72

Wiseman J, Cole DJA (1988) European legumes in diets for non-ruminants. In: Recent Advances in Animal Nutrition (W Haresign, DJA Cole, eds), Butterworths, Londres, Royaume-Uni, 13-37 